\title{
CLASS NUMBER IN CONSTANT EXTENSIONS OF FUNCTION FIELDS
}

\author{
JAMES R. C. LEITZEL
}

ABSTRACT. Let $F / K$ be a function field in one variable of genus $g$ having the finite field $K$ as exact field of constants. Suppose $p$ is a rational prime not dividing the class number of $F$. In this paper an upper bound is derived for the degree of a constant extension $E$ necessary to have $p$ occur as a divisor of the class number of the field $E$.

Throughout this paper the term "function field" will mean a function field in one variable whose exact field of constants is a finite field with $q$ elements.

Let $F / K$ be a function field. The order of the finite group of divisor classes of degree zero is the class number $h_{F}$. For $F / K$ of genus $g$, we use the notation of [2] and denote by $L(u)$ the polynomial numerator of the zeta function of $F$. It follows from the functional equation of the zeta function that

$$
\begin{aligned}
L(u)= & 1+a_{1} u+a_{2} u^{2}+\cdots \\
& +a_{g} u^{g}+q a_{g-1} u^{g+1}+\cdots+q^{g-1} a_{1} u^{2 g-1}+q^{g} u^{2 g}
\end{aligned}
$$

and $L(u) \in Z[u], Z$ the rational integers. Furthermore the class number $h_{F}=L(1)$. If $E / F$ is a constant field extension of degree $n$, then the polynomial numerator $L_{n}(u)$ of the zeta function for $E$ is given by

$$
L_{n}(u)=1+b_{1} u+\cdots+b_{g} u^{g}+q^{n} b_{g-1} u^{g+1}+\cdots+q^{n g} u^{2 g}
$$

where the coefficients $b_{j}(j=1, \cdots, g)$ are, with appropriate sign, the elementary symmetric functions of the $n$th powers of the reciprocals of the roots of (1). The genus of $E$ is the same as that of $F$ because $F$ is conservative.

In this paper we give an upper bound for the degree of a constant extension $E$ of $F$ necessary to have a predetermined prime $p$ occur as a

Received by the editors September 10, 1971 and, in revised form, March 12, 1972. AMS 1969 subject classifications. Primary 1078; Secondary 1278, 1435.

Key words and phrases. Zeta function, constant extension, reciprocal polynomials.

(c) American Mathematical Society 1972 
divisor of the class number $h_{E}$. Precisely, we prove

THEOREM 1. Let $F / K$ be a function field of genus $g$ and $p$ a rational prime. If $p \nmid h_{F}$ then $p \mid h_{E}$ for $E$ a constant extension of $F$ of degree $m$ where

(a) $m=f\left(p^{2 r(g)}-1\right)$ if $p \neq \operatorname{char} K$ and $f=\operatorname{ord} q(p)$.

(b) $m=p^{r(g)}-1$ if $p=$ char $K$ and $L(u) \not \equiv 1$ in $Z_{p}[u]$.

Here $r(g)$ denotes the least common multiple of the integers $1,2, \cdots, g$.

1. We collect here some results from the theory of equations. For $K$ a field, we say $f(x) \in K[x]$ is a reciprocal polynomial if and only if $f(x)=$ $x^{\operatorname{deg} f} f(1 / x)\left[1\right.$, Vol. 1, §32]. Observe that if $f(x)=a_{0}+a_{1} x+\cdots+x^{n}$ and $f(x)$ is a reciprocal polynomial then $a_{n-i}=a_{i}, i=1, \cdots,[n / 2]$, since necessarily $a_{0}=+1$.

Lemma 1. Let $K$ be a finite field, $f(x) \in K[x]$ a monic reciprocal polynomial of even degree $2 m$. Let $E$ be a splitting field for $f(x)$ over $K$, then $[E: K] \mid 2 r(m)$, where $r(m)$ is the least common multiple of the integers 1,2 , $\cdots, m$.

Proof. Suppose

$$
f(x)=x^{2 m}+a_{1} x^{2 m-1}+\cdots+a_{m} x^{m}+\cdots+a_{1} x+1 .
$$

Dividing by $x^{m}$ and combining terms we get

$$
\begin{aligned}
f(x) / x^{m}= & \left(x^{m}+1 / x^{m}\right)+a_{1}\left(x^{m-1}+1 / x^{m-1}\right) \\
& +\cdots+a_{m-1}(x+1 / x)+a_{m} .
\end{aligned}
$$

Set $z=x+1 / x$ and for nonnegative integers $s, W_{s}=x^{s}+1 / x^{s}$. It is easy to verify that $W_{s+1}=z W_{s}-W_{s-1}$. Substituting into (3) we get a polynomial $g(z)$ of degree $m$. Since $z=x+1 / x$ the roots of $f(x)$ can be obtained from the roots of $g(z)$ by solving quadratic polynomials. Since finite fields have cyclic galois groups we have from elementary field theory that $g(z)$ splits in an extension of degree at most $r(m)$. For a finite field there is a unique quadratic extension, so a splitting field $E$ for $f(x)$ has degree dividing $2 r(m)$.

Now let $K$ be arbitrary and $f(x) \in K[x]$ with degree $f=n$. Then if $\alpha_{1}$, $\cdots, \alpha_{n}$ are the roots of $f(x)$ in a splitting field the sums of the $k$ th powers of these roots are elements in $K$. In fact if we let $S_{k}=\sum_{i=1}^{n} \alpha_{i}^{k}$, then the following relations hold $[4$, p. 81$]$ :

$$
\begin{array}{ll}
S_{k}=S_{k-1} \sigma_{1}-S_{k-2} \sigma_{2}+\cdots+(-1)^{k+1} k \sigma_{k} & \text { for } k \leqq n, \\
S_{k}=S_{k-1} \sigma_{1}-S_{k-2} \sigma_{2}+\cdots+(-1)^{n+1} S_{k-n} \sigma_{n} & \text { for } k>n
\end{array}
$$

where $\sigma_{i}(i=1, \cdots, n)$ are the elementary symmetric functions of the roots. 
LEMMA 2. Let $Z$ denote the rational integers, $f(x) \in Z[x]$ a monic polynomial. Let $p$ be a rational prime and $f^{*}(x) \in Z_{p}[x]$ the image of $f(x)$ under the canonical homomorphism of $Z[x] \rightarrow Z_{p}[x]$. Let $S_{k}\left(S_{k}^{*}\right)$ denote the sum of the kth powers of the roots of $f(x)\left(f^{*}(x)\right)$. Then for all $k$ we have $S_{k} \equiv S_{k}^{*}(p)$.

Proof. Let $\sigma_{i}\left(\sigma_{i}^{*}\right), i=1, \cdots, \operatorname{deg} f$, denote the elementary symmetric functions of the roots of $f(x)\left(f^{*}(x)\right)$. Since the coefficients of $f(x)\left(f^{*}(x)\right)$ are, with appropriate sign, these elementary symmetric functions we have $\sigma_{i} \equiv \sigma_{i}^{*}(p)$ for all $i$ by definition. The conclusion then follows from the relations given in (4).

COROLlary 2.1. If $f(x) \in Z[x]$ is a monic polynomial of degree $2 m$ and $p$ a prime in $Z$ such that $f^{*}(x) \in Z_{p}[x]$ is a reciprocal polynomial we have

$$
S_{p^{2 r(m)}-1} \equiv 2 m(p) \text {. }
$$

Proof. By Lemma 1 if $\left[E: Z_{p}\right]=2 r(m)$ then $E$ contains a splitting field for $f^{*}(x)$. In $E$, every $\beta \neq 0$ satisfies $\beta^{p^{2 r(m)}-1}=1$. Therefore by Lemma 2 ,

$$
S_{p^{2 r(m)-1}} \equiv S_{p^{2 r(m)-1}}^{*} \equiv 2 m(p) .
$$

It is clear from (4) that the elementary symmetric functions of the roots of a polynomial can be expressed in terms of the $S_{k}$. In fact [1, Vol. 2, p. 39] if $f(x)=x^{n}+\sum_{r=1}^{n} a_{r} x^{n-r}$ then for $r=1, \cdots, n$ we have

$$
r ! a_{r}=(-1)^{r} \operatorname{det} A_{r}
$$

where $A_{r}$ is the $r \times r$ matrix given by

$$
A_{r}=\left(\begin{array}{ccccc}
S_{1} & 1 & 0 & \cdots & 0 \\
S_{2} & S_{1} & 2 & \cdots & 0 \\
\cdot & & & & \\
\cdot & & & \\
\cdot & & & \\
S_{r-1} & S_{r-2} & & \cdots & r-1 \\
S_{r} & S_{r-1} & & \cdots & S_{1}
\end{array}\right) .
$$

In the work that follows we will need to compute the determinant of matrices of the form (6) where the entries $S_{i}$ have particular values. All of these are of the general type described in the next result. 
LEMMA 3. Let $x, a, k$ be nonnegative integers with $k \mid x$, say $x=k y$. Let $A$ be the $r \times r$ matrix

$$
A=\left(\begin{array}{ccccc}
x a & k & 0 & \cdots & 0 \\
x a^{2} & x a & 2 k & \cdots & 0 \\
\cdot & & & & \\
\cdot & & & & \\
\cdot & & & & \\
x a^{r-1} & x a^{r-2} & & \cdots & (r-1) k \\
x a^{r} & x a^{r-1} & & \cdots & x a
\end{array}\right) ;
$$

then $\operatorname{det} A=k^{r} a^{r} \prod_{j=0}^{r-1}(y-j)$.

Proof. Simply use elementary column operations and cofactor expansions; i.e., begin by subtracting $a$ times column 2 from column 1 and then expand by cofactors of the resulting column 1 .

2. Proof of Theorem 1(a). Let $p$ be a prime and $F / K$ a function field of genus $g$. Since constant extensions are essentially unique, we first make the constant extension of degree $f=\operatorname{ord} q(p)$. Thus without loss of generality we assume that $F / K$ is a function field with $|K|=q \equiv 1(p)$ and $p \neq$ char $K$. Let $L(u)$ be the polynomial numerator of the zeta function of $F$. Because of our assumptions on $p$ and $q$ and the form (1) of $L(u)$ we see that $L^{*}(u) \in$ $Z_{p}[u]$ is a reciprocal polynomial of degree $2 g$. Hence from Corollary 2.1 we have, for $S_{n}$ denoting the sums of the $n$th powers of the reciprocals of the roots of $L(u)$,

$$
S_{k\left(p^{2 r(g)}-1\right)} \equiv 2 g(p), \quad k \in Z^{+}
$$

Let $m=p^{2 r(g)}-1$. The coefficients of $L_{m}(u)$ can be computed from (5); namely, $r ! b_{r}=(-1)^{r} \operatorname{det} A_{r}^{(m)}$, where

$$
A_{r}^{(m)}=\left(\begin{array}{ccccc}
S_{m} & 1 & & \cdots & 0 \\
S_{2 m} & S_{m} & 2 & \cdots & 0 \\
\cdot & & & & \\
\cdot & & & & \\
\cdot & & & \\
S_{r m} & S_{(r-1) m} & & \cdots & S_{m}
\end{array}\right) .
$$

Using $S_{k m} \equiv 2 g(p)$ and Lemma 3 with $x=2 g, a=k=1$, we deduce

$$
b_{r} \equiv(-1)^{r}\left(\begin{array}{c}
2 g \\
r
\end{array}\right)(p)
$$


Moreover

$$
h_{E}=L_{m}(1)=1+q^{m g}+\sum_{i=1}^{g-1}\left(1+q^{m(g-i)}\right) b_{i}+b_{g} .
$$

Substituting from (8) we get

$$
h_{E} \equiv 2+2 \sum_{i=1}^{g-1}(-1)^{i}\left(\begin{array}{c}
2 g \\
i
\end{array}\right)+(-1)^{g}\left(\begin{array}{c}
2 g \\
g
\end{array}\right)(p) .
$$

Observing that $(-1)^{i}\left(\begin{array}{c}2 g \\ i\end{array}\right)=(-1)^{2 g-i}\left(\begin{array}{c}2 \rho \\ 2 g-i\end{array}\right)$ we conclude

$$
h_{E} \equiv \sum_{i=0}^{2 g}(-1)^{i}\left(\begin{array}{c}
2 g \\
i
\end{array}\right) \equiv 0(p) .
$$

3. Proof of Theorem 1(b). Suppose now $F / K$ is a function field of genus $g$, and $p$ a prime with $p=\operatorname{char} K$. Let $L(u)$ as given by (1) denote the polynomial numerator of the zeta function of $F$. Assume that $L^{*}(u) \not \equiv 1$ in $Z_{p}[u]$ and set $t=\max \left\{j \mid\right.$ such that $\left.a_{j} \neq \equiv(p)\right\}$. Clearly $1 \leqq t \leqq g$. Consequently $L^{*}(u)$ is a polynomial of degree $t$ and therefore splits in the extension of $Z_{p}$ of degree $r(t)$. As before denoting by $S_{n}^{*}$ the sum of the $n$th powers of the reciprocals of the roots of $L^{*}(u)$, we have, as in Corollary 2.1,

$$
S_{k\left(p^{r(t)}-1\right)} \equiv t(p), \quad k \in Z^{+} .
$$

If $E$ is the constant extension of degree $m=p^{r(t)}-1$, then to compute $h_{E}$ we need the coefficients $b_{i}(i=1, \cdots, g)$ of $L_{m i}(u)$ as given by (2).

From Lemma 3 with $x=t, a=k=1$ we see

Then

$$
\begin{array}{ll}
b_{j} \equiv(-1)^{j}\left(\begin{array}{l}
t \\
j
\end{array}\right)(p), & j=1, \cdots, t, \\
b_{j} \equiv 0(p), & j=t+1, \cdots, g .
\end{array}
$$

$$
h_{E}=L_{m}(1)=1+q^{m g}+\sum_{i=1}^{g-1} b_{i}\left(1+q^{m(g-i)}\right)+b_{g}
$$

gives, after substitution from (12) and $q \equiv 0(p)$,

$$
h_{E} \equiv 1+\sum_{i=1}^{t}(-1)^{i}\left(\begin{array}{l}
t \\
i
\end{array}\right)(p) \text {, }
$$

i.e., $h_{E} \equiv \sum_{i=0}^{t}(-1)^{i}\left(\begin{array}{l}t \\ i\end{array}\right) \equiv 0(p)$. Since $p^{r(t)}-1 \mid p^{r(g)}-1$ we have Theorem $1(b)$.

Note. If $L(u) \not \equiv 1(p)$ we can extend the argument to produce a value $m^{\prime}$ such that the constant extension $E / F$ of degree $m^{\prime}$ has $h_{E}$ divisible by 
$p^{s}, s \geqq 1$. From Leitzel [3, Theorem 2], we have if $p \mid h_{M}$ and $T / M$ is the constant extension of degree $p$ then $h_{T}$ is divisible by at least $p^{2}$, since the $p$-rank of $h_{M}$ is larger than one. Thus $h_{E}$ is divisible by $p^{s}, s \geqq 1$, if $E / F$ is the constant extension of degree $m^{\prime}=m p^{s-1}$, where $m$ is the value determined in the above Theorem 1.

I am indebted to the referee for indicating the following more direct proof of this extended result: We have $L(u)=\prod_{i=1}^{2 g}\left(1-v_{i} u\right)$ where the $w_{i}$ are algebraic integers. Let $L^{\prime \prime}$ be the splitting field of $L(u)$ over $Q$. Let $P$ be a prime of $L^{\prime \prime}$ dividing $p$. Then $P \nmid x_{i}$ for at least one $i$ (since otherwise $L(u) \equiv 1(\bmod P)$, and thus also $(\bmod p))$. Let $L^{\prime}=Q\left(w_{i}^{\prime}\right)$ and $P^{\prime}$ the prime of $L^{\prime}$ divisible by $P$. Then $e^{\prime} f^{\prime} \leqq 2 g$ where $e^{\prime}$ and $f^{\prime}$ are ramification index and residue class degree of $P^{\prime}$ over $Q$. Also, the order of the multiplicative group of the residue class ring of the integers in $L^{\prime}$ modulo $P^{\prime} e^{\prime}(s-1)+1$ is $m=\left(p^{f^{\prime}}-1\right) p^{e^{\prime} f^{\prime}(s-1)}$. Thus $x_{i}^{m} \equiv 1\left(\bmod P^{\prime} e^{\prime}(s-1)+1\right)$ and so $h_{E}=L_{m}(1) \equiv 0$ $\left(\bmod P^{\prime} e^{\prime}(s-1)+1\right)$. But then, $h_{E} \equiv 0\left(\bmod p^{s}\right)$. Arguments similar to those of Theorem 1 can be applied to show that $m$ can be taken as $f\left(p^{2 r(g)}-1\right) p^{2 g(s-1)}$ in case (a) (where $p \nmid q$ ) and $\left(p^{r(g)}-1\right)^{p g(s-1)}$ in case (b) (where $p \mid q$ ).

4. An additional comment. In $\S 3$ we discussed the situation where $F / K$ is a function field of genus $g, p=\operatorname{char} K$, and $L^{*}(u) \not \equiv 1$ in $Z_{p}[u]$. In this section we discuss the case $L^{*}(u) \equiv 1$ in $Z_{p}[u]$.

Let $F / K$ be a function field of genus $g$ and $p$ a prime. Suppose $L(u)$, the polynomial numerator of the zeta function of $F$ as given by (1), satisfies the condition

$$
a_{i} \equiv 0(p), \quad i=1, \cdots, g .
$$

Then $L^{*}(u)=1+q^{g} u^{2 g}$ in $Z_{p}[u]$ if $p \neq \operatorname{char} K$ and $L^{*}(u) \equiv 1$ in $Z_{p}[u]$ if $p=$ char $K$. For a function field satisfying the condition (14) we give an explicit congruence relation for the class number $h_{E}$ of any constant extension $E / F$. This is contained in

THEOREM 2. Let $F / K$ be a function field of genus $g$ and $p$ a prime. Suppose $L^{*}(u)=1+q^{g} u^{2 g}$ in $Z_{p}[u]$ and $E / F$ is a constant extension of degree $m$. Then if $d=\operatorname{gcd}(m, 2 g)$ we have

$$
h_{E} \equiv\left[1-(-1)^{m / d} q^{g m / d}\right]^{d}(p) .
$$

Proof. Let $S_{n}$ again denote the sum of the $n$th powers of the reciprocals of the roots of $L(u)$. From our assumption on $L(u)$ and the relations of (4) we deduce

$$
\begin{array}{ll}
S_{n} \equiv 0(p) & \text { if } 2 g \nmid n, \\
S_{n} \equiv(-1)^{k} 2 g q^{k g}(p) & \text { if } n=2 g k .
\end{array}
$$

To compute $h_{E^{\prime}}$ for $E / F$ a constant extension of degree $m$ it is necessary to determine the coefficients of $L_{m}(u)$. These all require the computation of 
the determinant of a matrix of the type (7). Because of the relations (16), nonzero entries occur only when $j m \equiv 0(2 g), j=1, \cdots, r$. If $d=\operatorname{gcd}(m, 2 g)$ and $m=t d, 2 g=k d$, then the values of $j$ which yield nonzero entries are precisely $l k$ for $1 \leqq l \leqq[d / 2]$. Thus using this observation we can express the coefficients as

$$
b_{l k} \equiv \frac{(-1)^{2 l k-l}}{(l k) !} \frac{(l k-1) !}{k 2 k \cdots(l-1) k}
$$

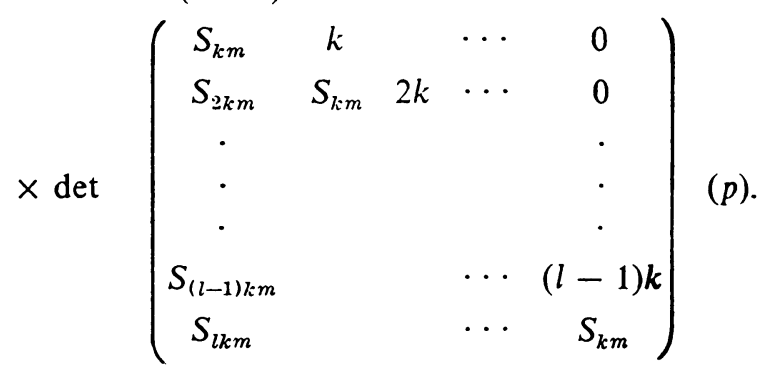

Here we have used (16) and cofactor expansions along rows to get the final form. Now apply Lemma 3 with $x=2 g=k d$ and $a=\left(-q^{g}\right)^{m / d}$. We have then

$$
\begin{aligned}
b_{l k} & \equiv \frac{(-1)^{2 l k-l}}{l ! k^{l}} k^{l}\left(-q^{g}\right)^{m l / d} \prod_{j=0}^{l-1}(d-j)(p) \\
& \equiv(-1)^{2 l k-l}\left(-q^{g}\right)^{m l / d}\left(\frac{d}{l}\right)(p) .
\end{aligned}
$$

Substituting this information in

we find, for odd $d$,

$$
h_{E}=L_{m}(1)=1+q^{m \sigma}+\sum_{i=1}^{a-1} b_{i}\left(1+q^{m(g-i)}\right)+b_{g}
$$

or

$$
h_{E} \equiv 1+q^{m g}+\sum_{l=1}^{[d / 2]} b_{k l}\left(1+q^{m(g-k l)}\right)(p)
$$

Since

$$
h_{E} \equiv 1+q^{m g}+\sum_{l=1}^{[d / 2]}\left(1+q^{m(g-k l)}\right)(-1)^{2 l k-l}\left(-q^{g}\right)^{m l / d}\left(\frac{d}{l}\right)(p) .
$$

$$
q^{m(g-k l)}(-1)^{l}\left(-q^{g}\right)^{m l / d}\left(\frac{d}{l}\right)=(-1)^{d-l}\left(\frac{d}{d-l}\right)\left(-q^{g}\right)^{m(d-l) / d}
$$

and $m+d \equiv 0(2)$ this can be rewritten as

$$
h_{E} \equiv \sum_{l=0}^{d}(-1)^{l}\left(\frac{d}{l}\right)\left((-1)^{m / d} q^{m g / d}\right)^{l}(p)
$$


If $d$ is even a similar argument leads to the same formula. Hence

$$
h_{E} \equiv\left[1-(-1)^{m / d} q^{n g / d}\right]^{d}(p) .
$$

Corollary 1. If $\operatorname{gcd}(m, 2 g)=1$, then $h_{E} \equiv 1+q^{m g}(p)$.

Proof. $(m, 2 g)=1$ forces $d=1$ and $m \equiv 1$ (2).

COROLlaRY 2. If $2 g \mid m$, then for $m=2 g t$ we have $h_{k} \equiv$ $\left[1-(-1)^{t} q^{g t}\right]^{2 g}(p)$.

COROLlary 3. If $p=\operatorname{char} K$ and $L(u) \equiv 1$ in $Z_{p}[u]$ then $p \nmid h_{k}$ for any constant extension $E / F$.

Proof. Clearly $h_{E} \equiv 1(p)$ in this case.

\section{BIBLIOGRAPHY}

1. W. S. Burnside and A. W. Panton, The theory of equations: With an introduction to the theory of binary algebraic forms. Vols. 1, 2, Dover, New York, 1960. MR 22 \#6784.

2. M. Eichler, Introduction to the theory of algebraic numbers and functions, Birkhäuser, Basel, 1963; English transl., Pure and Appl. Math., vol. 23, Academic Press, New York, 1966. MR 29 \#5821; MR 35 \#160.

3. J. R. C. Leitzel, Galois cohomology and class number in constant extensions of algebraic function fields, Proc. Amer. Math. Soc. 22 (1969), 206-208. MR 39 \#4126.

4. B. L. van der Waerden, Moderne Algebra. Vol. 1, 2nd rev. ed., English transl., Ungar, New York, 1953.

Department of Mathematics, Ohio State University, Columbus, Ohio 43210 